\title{
Continuidad de funciones basadas en reordenamientos de $\beta$-expansiones de un número
}

| Continuity of functions based on rearrangements of a $\beta$-expansion of a number |

\author{
Andrés Merino \\ aemerinot@puce.edu.ec \\ Escuela de Ciencias Físicas y Matemática \\ Facultad de Ciencias Exactas y Naturales \\ Pontificia Universidad Católica del Ecuador \\ Quito - Ecuador
}

(D) Jonathan Ortiz-Castro

jonathan.ortizc@epn.edu.ec

Facultad de Ciencias

Escuela Politécnica Nacional

Quito - Ecuador

Resumen: Las funciones dadas por reordenaciones de $\beta$-expansiones de un número suelen ser presentadas como ejemplos de variables aleatorias en la teoría de la probabilidad, sin embargo, no se realiza un estudio a profundidad de este tipo de funciones ni se suele presentar una demostración rigurosa de que, en efecto, son variables aleatorias. En el presente trabajo, se da una demostración del resultado original de que este tipo de funciones son continuas en casi todas partes, de lo cual se deduce que son variables aleatorias. Además, se presentan demostraciones originales y directas de algunas propiedades conocidas de las $\beta$-expansiones de los números entre 0 y 1 ; entre estas, se establece condiciones para que un número tenga $\beta$-expansión única y también se prueba que si dos números tienen $\beta$-expansión única y son suficientemente cercanos, entonces sus $\beta$-expansiones coinciden hasta cierto índice. Finalmente, se presenta una caracterización original sobre los puntos de continuidad de funciones dadas por reordenaciones estrictamente crecientes.

Palabras Clave: variables aleatorias, $\beta$-expansiones, continuidad, teoría de la medida

\begin{abstract}
The functions given by rearrangements of $\beta$-expansions of a number are usually presented as examples of random variables in probability theory, however, an in-depth study of this type of functions is not carried out nor is a rigorous demonstration that they are indeed random variables. In this work it is proved a proof of the original result that these types of functions are continuous almost everywhere, and so they are random variables. In addition, it is presented original and direct proofs of the most known properties of $\beta$-expansions in $[0,1]$; for example: conditions for that a number has unique $\beta$-expansion, and it is proved that if two number, with unique $\beta$-expansion, are close enough, then their $\beta$-expansions match up to a certain index. Finally, an original proof is presented of the points of continuity of functions given by strictly increasing rearrangements.
\end{abstract}

Keywords: random variables, $\beta$-expansions, continuity, measure theory 


\section{Introducción}

La demostración de la existencia de una sucesión de variables aleatorias independientes no es un problema trivial (Borovkov, 2013, p. 56), pero es de gran importancia, ya que existe un amplio número de resultados de la teoría de la probabilidad que utilizan este tipo de sucesiones (ver Chung y Zhong (2001); Borovkov (2013)). En la literatura se pueden encontrar diversas demostraciones de la existencia de este tipo de variables aleatorias, sin embargo, la mayoría de estas demostraciones no son constructivas o utilizan resultados clásicos de Kolmogorov o Caratheodory basados en medidas productos o extensión de medidas. Por otro lado, existe una demostración constructiva y que no requiere la utilización de resultados adicionales, ver Billingsley (2012); Kallenberg (2002); Williams (1991); esta demostración utiliza la expansión binaria de los números reales, en específico, Williams (1991) lo plantea de la siguiente manera ${ }^{1}$ : para $x \in[0,1]$, se toma $\left(x_{n}\right)_{n \in \mathbb{N}}$ tal que

$$
x=\frac{x_{1}}{2^{1}}+\frac{x_{2}}{2^{2}}+\frac{x_{3}}{2^{3}}+\frac{x_{4}}{2^{4}}+\cdots
$$

y se define las funciones

$$
\begin{aligned}
& \varphi_{1}(x)=\frac{x_{1}}{2^{1}}+\frac{x_{3}}{2^{2}}+\frac{x_{6}}{2^{3}}+\frac{x_{10}}{2^{4}}+\cdots, \\
& \varphi_{2}(x)=\frac{x_{2}}{2^{1}}+\frac{x_{5}}{2^{2}}+\frac{x_{9}}{2^{3}}+\frac{x_{14}}{2^{4}}+\cdots, \\
& \varphi_{3}(x)=\frac{x_{4}}{2^{1}}+\frac{x_{8}}{2^{2}}+\frac{x_{13}}{2^{3}}+\frac{x_{19}}{2^{4}}+\cdots, \\
& \varphi_{4}(x)=\frac{x_{7}}{2^{1}}+\frac{x_{12}}{2^{2}}+\frac{x_{18}}{2^{3}}+\frac{x_{25}}{2^{4}}+\cdots,
\end{aligned}
$$

Se puede apreciar que se utiliza una especie de zigzag de Cantor para la colocación de los subíndices. Con esto, Williams establece que $\left(\varphi_{n}\right)_{n \in \mathbb{N}}$ es una sucesión de variables aleatorias independientes. Las sucesiones planteadas por Billingsley (2012) y Kallenberg (2002) son similares, pero en ninguno de los tres textos se demuestra que en efecto las funciones que definen son variables aleatorias y únicamente se centran en demostrar su independencia.

En este trabajo, con la finalidad de llenar el vacío dejado por las demostraciones de Billingsley, Kallenberg, Williams, entre otros, se presentará una demostración original y a detalle, de que una familia de funciones, de las cuales las funciones definidas en las ecuaciones (1) son un caso particular, es una familia de funciones continuas en casi todas partes (es decir, que el conjunto de discontinuidades tiene medida nula, ver Shirali (2018, p. 118)) y, por ende, variables aleatorias (Gentili, 2020, p. 242). En específico, se demostrará a detalle que, dada una función $\psi: \mathbb{N}^{*} \rightarrow \mathbb{N}^{*}$ (que llamaremos reordenamiento), si para $x \in[0,1]$, se toma $\left(x_{n}\right)_{n \in \mathbb{N}}$ una $\beta$-expansión de $x$ y se define la función

$$
F_{\psi}(x)=\frac{x_{\psi(1)}}{\beta^{1}}+\frac{x_{\psi(2)}}{\beta^{2}}+\frac{x_{\psi(3)}}{\beta^{3}}+\frac{x_{\psi(4)}}{\beta^{4}}+\cdots,
$$

entonces $F_{\psi}$ es continua en casi todas partes $y$, por lo tanto, una variable aleatoria.

Como se puede ver, el tipo de funciones definidas por la ecuación (2) están relacionadas con la $\beta$ expansión de un número, por esta razón, se presentan algunas notaciones y resultados acerca de las expansiones de un número con la finalidad de expresar estas funciones de una manera precisa y con notación contemporánea.

Se utilizan las siguientes notaciones a lo largo del trabajo. Se toma $\mathbb{N}^{*}=\mathbb{N} \backslash\{0\} ;$ dados $a, b \in \mathbb{Z}$ con $a \leq b$, se denota $\llbracket a, b \rrbracket=[a, b] \cap \mathbb{Z}$, es decir, el conjunto de todos los números enteros comprendidos entre a y b. Además, se denota por $\mathcal{J}$ al intervalo $] 0,1[\subseteq \mathbb{R}$; por otro lado, si $x \in \mathbb{R},\lfloor x\rfloor$ representará la

\footnotetext{
${ }^{1}$ Se presenta aquí el ejemplo tal y como consta en el texto original.
} 
parte entera de $x$, es decir, el mayor número entero menor o igual a $x$. Finalmente, dado un conjunto $A, A^{\mathbb{N}^{*}}$ representa el conjunto de todas las sucesiones en $A$ (que empiezan su numeración en 1 ).

Ahora, dados $\beta \in \mathbb{N}$ tal que $\beta>1$ y $x \in \mathcal{J} \cup\{0,1\}$, una $\beta$-expansión de $x$ es una sucesión $\left(x_{\mathfrak{n}}\right)_{\mathfrak{n} \in \mathbb{N}}$ de $\llbracket 0, \beta-1 \rrbracket$ tal que

$$
x=\sum_{n=1}^{+\infty} \frac{x_{n}}{\beta^{n}} .
$$

Se tiene que todo elemento de $\mathcal{J} \cup\{0,1\}$ posee al menos una $\beta$-expansión (la demostración original de este hecho puede encontrarse en Rényi (1957)). Con esto, definiendo la función

$$
\begin{aligned}
\mathrm{T}_{\beta}: \llbracket 0, \beta-1 \rrbracket^{\mathbb{N}^{*}} & \longrightarrow \mathcal{J} \cup\{0,1\} \\
\left(x_{n}\right)_{n \in \mathbb{N}} & \longmapsto \sum_{n=1}^{+\infty} \frac{x_{n}}{\beta^{n}},
\end{aligned}
$$

se tiene que esta función es sobreyectiva más no inyectiva.

Por otro lado, dado $x \in \mathcal{J}$, existe una única $\beta$-expansión $\left(x_{n}\right)_{n \in \mathbb{N}}$ tal que

$$
\left(\forall k \in \mathbb{N}^{*}\right)(\exists m>k)\left(x_{m} \neq \beta-1\right),
$$

es decir, una $\beta$-expansión que no tiene una cola de términos $\beta-1$. Por lo tanto, si definimos

$$
\mathcal{D}_{\beta}=\left\{\left(x_{\mathfrak{n}}\right)_{\mathfrak{n} \in \mathbb{N}} \in \llbracket 0, \beta-1 \rrbracket^{\mathbb{N}^{*}}:\left(\forall k \in \mathbb{N}^{*}\right)(\exists m>k)\left(x_{\mathfrak{m}} \neq \beta-1\right)\right\} \backslash\left\{(0)_{\mathfrak{n} \in \mathbb{N}}\right\},
$$

se tiene que la función

$$
\left.\mathrm{T}_{\beta}\right|_{\mathcal{D}_{\beta}}: \mathcal{D}_{\beta} \rightarrow \mathcal{J}
$$

es biyectiva. Con esto, se nombra $E^{\beta}$ a la inversa de $\left.T_{\beta}\right|_{\mathcal{D}_{\beta}}$, es decir,

$$
E^{\beta}: \mathcal{J} \rightarrow \mathcal{D}_{\beta}
$$

es la función tal que asigna a cada elemento de $\mathcal{J}$ su única $\beta$-expansión que no posee una cola de números $\beta-1$. Además, para cada $n \in \mathbb{N}^{*}$, se define $E_{n}^{\beta}: \mathcal{J} \rightarrow \llbracket 0, \beta-1 \rrbracket$ a la función que asigna a cada $x \in \mathcal{J}$ la $n$-ésima componente de $E^{\beta}(x)$ (en caso de no presentar ambigüedad, se la denota simplemente por $E_{n}$ ), es decir, le asigna la n-ésima componente de su $\beta$-expansión que no posee una cola de números $\beta-1$. Así, se tiene que, para todo $x \in \mathcal{J}$,

$$
x=\sum_{n=1}^{+\infty} \frac{E_{n}^{\beta}(x)}{\beta^{n}}=\sum_{n=1}^{+\infty} \frac{E_{n}(x)}{\beta^{n}} .
$$

Finalmente, se define

$$
\varepsilon_{\beta}=\left\{\left(x_{n}\right)_{n \in \mathbb{N}} \in \llbracket 0, \beta-1 \rrbracket^{\mathbb{N}^{*}}:\left(\forall k \in \mathbb{N}^{*}\right)(\exists m>k)\left(x_{m} \neq 0\right)\right\} \backslash\left\{(\beta-1)_{n \in \mathbb{N}}\right\},
$$

así, $\mathcal{D}_{\beta} \cap \mathcal{E}_{\beta}$ es el conjunto de sucesiones que no tienen ni cola de números 0 ni de números $\beta-1$. Con esto, se define $\mathcal{J}=T_{\beta}\left(\mathcal{D}_{\beta} \cap \mathcal{E}_{\beta}\right)$, es decir, $\mathcal{J}$ es el conjunto de todos los elementos de $\mathcal{J}$ con $\beta$-expansión sin cola de números 0 ni de números $\beta-1$.

Con la finalidad de presentar un trabajo autocontenido, en la sección 2, se presenta la demostración de existencia y las condiciones para unicidad de la $\beta$-expansión de los elementos de $\mathcal{J}$, además, se dan algunos resultados que serán de utilidad en la sección posterior. En la sección 3, se presentan algunas propiedades de las funciones que se basan en reordenamientos de las $\beta$-expansiones de un número, adicionalmente, se demuestra que funciones análogas a las dadas en Williams (1991) son continuas en casi todas partes. Todas las proposiciones y teoremas presentados en este trabajo, salvo las Proposiciones 1 y 2, no se hallan en la literatura especializada y, junto con todas las demostraciones presentadas, son originales de los autores. 


\section{Propiedades de la $\beta$-expansión}

El resultado de que todo elemento de $\mathcal{J} \cup\{0,1\}$ posee al menos una $\beta$-expansión es ampliamente conocido y su demostración original puede ser encontrada en Rényi (1957), sin embargo, en general, la demostración de este hecho no es directa, sino que se la presenta como un corolario de resultados más generales. Por esta razón, y para la completitud de este documento, se presenta aquí una demostración centrada en el caso específico de $\beta$-expansiones con $\beta$ un número natural mayor que 1 , utilizando la notación contemporánea dada en la introducción. A partir de este punto, siempre se considera $\beta \in \mathbb{N}$ tal que $\beta>1$.

\section{Proposición 1}

Sea $x \in \mathcal{J}$. Se tiene que existe una sucesión $\left(x_{\mathfrak{n}}\right)_{\mathfrak{n} \in \mathbb{N}}$ de $\llbracket 0, \beta-1 \rrbracket$ tal que

$$
x=\sum_{n=1}^{+\infty} \frac{x_{n}}{\beta^{n}} .
$$

Demostración. Se va a definir $\left(x_{n}\right)_{n \in \mathbb{N}}$ de manera recursiva. Se toma $x_{1}=\lfloor\beta x\rfloor y$, para cada entero $n \geq 2$,

$$
x_{n}=\left\lfloor\beta^{n} x-\sum_{j=1}^{n-1} \beta^{n-j} x_{j}\right\rfloor .
$$

Se tiene que $\left(x_{n}\right)_{\mathfrak{n} \in \mathbb{N}}$ es una $\beta$-expansión de $x$, en efecto, se nota que

$$
\begin{aligned}
0 \leq x<1 & \Longleftrightarrow 0 \leq \beta x<\beta \\
& \Longleftrightarrow 0 \leq\lfloor\beta x\rfloor<\beta \\
& \Longleftrightarrow x_{1} \in \llbracket 0, \beta-1 \rrbracket .
\end{aligned}
$$

Además, para cada entero $n \geq 2$, se tiene que

$$
\begin{aligned}
\beta^{n-1} x-\sum_{j=1}^{n-1} \beta^{n-1-j} x_{j} & =\left(\beta^{n-1} x-\sum_{j=1}^{n-2} \beta^{n-1-j} x_{j}\right)-x_{n-1} \\
& =\left(\beta^{n-1} x-\sum_{j=1}^{n-2} \beta^{n-1-j} x_{j}\right)-\left\lfloor\beta^{n-1} x-\sum_{j=1}^{n-2} \beta^{n-1-j} x_{j}\right\rfloor,
\end{aligned}
$$

por lo tanto,

$$
\begin{aligned}
0 \leq \beta^{n-1} \chi-\sum_{j=1}^{n-1} \beta^{n-1-j} x_{j}<1 & \Longleftrightarrow 0 \leq \beta^{n} \chi-\sum_{j=1}^{n-1} \beta^{n-j} x_{j}<\beta \\
& \Longleftrightarrow 0 \leq x_{n}<\beta \\
& \Longleftrightarrow x_{n} \in \llbracket 0, \beta-1 \rrbracket .
\end{aligned}
$$


Así, $\left(x_{n}\right)_{n \in \mathbb{N}}$ es una sucesión de $\llbracket 0, \beta-1 \rrbracket$. Por otro lado, dado un entero $n \geq 2$, se tiene que

$$
\begin{aligned}
x-\sum_{j=1}^{n} \frac{x_{j}}{\beta^{j}} & =\frac{\beta^{n} x-\sum_{j=1}^{n} \beta^{n-j} x_{j}}{\beta^{n}} \\
& =\frac{\left(\beta^{n} x-\sum_{j=1}^{n-1} \beta^{n-j} x_{j}\right)-x_{n}}{\beta^{n}} \\
& =\frac{\left(\beta^{n} x-\sum_{j=1}^{n-1} \beta^{n-j} x_{j}\right)-\left\lfloor\beta^{n} x-\sum_{j=1}^{n-1} \beta^{n-j} x_{j}\right\rfloor}{\beta^{n}},
\end{aligned}
$$

de donde

$$
0 \leq x-\sum_{j=1}^{n} \frac{x_{j}}{\beta^{j}} \leq \frac{1}{\beta^{n}},
$$

luego, tomando límite cuando $n \rightarrow+\infty$, se sigue que

$$
x=\sum_{n=1}^{+\infty} \frac{x_{n}}{\beta^{n}} .
$$

Con esto, se tiene que todo elemento de $\mathcal{J}$ posee al menos una $\beta$-expansión, sin embargo, es conocido que, en general, esta no es única; por ejemplo:

$$
\frac{1}{\beta}=\frac{1}{\beta^{1}}+\frac{0}{\beta^{2}}+\frac{0}{\beta^{3}}+\cdots \quad \text { y } \quad \frac{1}{\beta}=\frac{0}{\beta^{1}}+\frac{\beta-1}{\beta^{2}}+\frac{\beta-1}{\beta^{3}}+\cdots
$$

A pesar de esto, se puede demostrar que todo elemento de J posee a lo más dos $\beta$-expansiones en una de las cuales se tiene cola de números 0 y en la otra, cola de números $\beta-1$. Para demostrar esto, primero se nota que una forma de comparar dos $\beta$-expansiones $\left(x_{n}\right)_{n \in \mathbb{N}}$ y $\left(y_{n}\right)_{n \in \mathbb{N}}$ es tomar la diferencia entre los números que representan, es decir, analizar

$$
\sum_{n=1}^{+\infty} \frac{x_{n}-y_{n}}{\beta^{n}}
$$

así, esto recae en analizar el comportamiento de la sucesión $\left(x_{n}-y_{n}\right)_{\mathfrak{n} \in \mathbb{N}}$, la cual toma valores en $\llbracket-\beta+1, \beta-1 \rrbracket$; por esta razón, se demuestran resultados preliminares sobre las sucesiones de $\llbracket-\beta+$ $1, \beta-1 \rrbracket$ que serán de utilidad para la demostración de los resultados centrales como las Proposiciones 3 y 4 , por ejemplo, el siguiente lema.

Lema 1 Sea $\left(z_{n}\right)_{n \in \mathbb{N}}$ una sucesión en $\llbracket-\beta+1, \beta-1 \rrbracket$ tal que

$$
\sum_{n=1}^{+\infty} \frac{z_{n}}{\beta^{n}}=1 .
$$

Se tiene que $z_{m}=\beta-1$ para todo $m \in \mathbb{N}^{*}$. 
Demostración. Sea $m \in \mathbb{N}^{*}$, se tiene que

$$
\begin{aligned}
1=\sum_{n=1}^{+\infty} \frac{z_{n}}{\beta^{n}} & =\left(\sum_{\substack{n=1 \\
n \neq m}}^{+\infty} \frac{z_{n}}{\beta^{n}}+\frac{\beta-1}{\beta^{m}}\right)+\left(\frac{z_{m}}{\beta^{m}}-\frac{\beta-1}{\beta^{m}}\right) \\
& \leq \sum_{n=1}^{+\infty} \frac{\beta-1}{\beta^{n}}+\left(\frac{z_{m}}{\beta^{m}}-\frac{\beta-1}{\beta^{m}}\right) \\
& =1+\left(\frac{z_{m}}{\beta^{m}}-\frac{-\beta+1}{\beta^{m}}\right),
\end{aligned}
$$

por lo tanto,

$$
0 \leq \frac{z_{m}}{\beta^{m}}-\frac{\beta-1}{\beta^{m}}
$$

de donde $z_{m} \geq \beta-1$; como $z_{m} \in \llbracket-\beta+1, \beta-1 \rrbracket$, concluimos que $z_{m}=\beta-1$.

Corolario 1 coro1 Sean $m \in \mathbb{N}^{*}$ y $\left(z_{n}\right)_{n \in \mathbb{N}}$ una sucesión en $\llbracket-\beta+1, \beta-1 \rrbracket$ tal que

$$
\sum_{n=m}^{+\infty} \frac{z_{n}}{\beta^{n}}=\frac{1}{\beta^{m-1}}
$$

Se tiene que $z_{n}=\beta-1$ para todo $n \geq m$.

Demostración. Se nota que

$$
1=\beta^{m-1} \sum_{n=m}^{+\infty} \frac{z_{n}}{\beta^{n}}=\sum_{n=m}^{+\infty} \frac{z_{n}}{\beta^{n-m+1}}=\sum_{n=1}^{+\infty} \frac{z_{n+m}}{\beta^{n}},
$$

por el resultado anterior, se tiene que $z_{n+m}=\beta-1$ para todo $n \in \mathbb{N}^{*}$, es decir, $z_{n}=\beta-1$ para todo $n \geq m$.

Con esto, se procede a la demostración de que todo elemento de J posee a lo más dos $\beta$-expansiones.

\section{Proposición 2}

Sean $x \in \mathcal{J},\left(x_{n}\right)_{n \in \mathbb{N}} y\left(y_{n}\right)_{n \in \mathbb{N}}$ dos $\beta$-expansiones de $x$ distintas. Se tiene que existe $m \in \mathbb{N}^{*}$ tal que $x_{n}=y_{n}$ para todo $n \in \llbracket 1, m-1 \rrbracket,\left|x_{m}-y_{m}\right|=1 y$

$$
x_{n}=0 \quad y \quad y_{n}=\beta-1
$$

o

$$
x_{n}=\beta-1 \quad y \quad y_{n}=0
$$

para todo $n>m$.

Demostración. Como las sucesiones son diferentes, se toma

$$
m=\min \left\{n \in \mathbb{N}^{*}: x_{n} \neq y_{n}\right\}
$$


Es claro que $x_{n}=y_{n}$ para cada $n \in \llbracket 1, m-1 \rrbracket$, con esto, dado que ambas sucesiones son $\beta$-expansiones de $x$, se tiene que

$$
\sum_{n=m}^{+\infty} \frac{x_{n}}{\beta^{n}}=\sum_{n=m}^{+\infty} \frac{y_{n}}{\beta^{n}}
$$

de donde

$$
\frac{x_{m}-y_{m}}{\beta^{m}}=\sum_{n=m+1}^{+\infty} \frac{y_{n}-x_{n}}{\beta^{n}} .
$$

Como

$$
-\frac{1}{\beta^{m}}=\sum_{n=m+1}^{+\infty} \frac{-\beta+1}{\beta^{n}} \leq \sum_{n=m+1}^{+\infty} \frac{y_{n}-x_{n}}{\beta^{n}} \leq \sum_{n=m+1}^{+\infty} \frac{\beta-1}{\beta^{n}}=\frac{1}{\beta^{m}},
$$

se sigue que $-1 \leq x_{m}-y_{m} \leq 1$. Dado que $x_{m}$ y $y_{m}$ son enteros distintos, se sigue que $\left|x_{m}-y_{m}\right|=1$. Con esto, se tienen dos casos:

- Si $x_{m}-y_{m}=-1$, de la igualdad (3), se tiene que

$$
\sum_{n=m+1}^{+\infty} \frac{x_{n}-y_{n}}{\beta^{n}}=1
$$

de donde, por el resultado anterior, se sigue que $x_{n}-y_{n}=\beta-1$ para todo $n>m$, por lo tanto, como $x_{n}, y_{n} \in \llbracket 0, \beta-1 \rrbracket$ para todo $n>m$, concluimos que $x_{n}=\beta-1$ y $y_{n}=0$ para todo $n>m$.

- Si $x_{m}-y_{m}=1$, de la igualdad (3), se tiene que

$$
\sum_{n=m+1}^{+\infty} \frac{y_{n}-x_{n}}{\beta^{n}}=1
$$

de donde, por el resultado anterior, se sigue que $y_{n}-x_{n}=\beta-1$ para todo $n>m$, por lo tanto, como $x_{n}, y_{n} \in \llbracket 0, \beta-1 \rrbracket$ para todo $n>m$, concluimos que $y_{n}=\beta-1$ y $x_{n}=0$ para todo $n>m$.

Con este resultado y notando que todo elemento de J que posea una $\beta$-expansión con cola de números 0 también posee otra con cola de números $\beta-1$ y viceversa, quedan caracterizados los elementos de J que tienen $\beta$-expansión única. Así, el conjunto $\mathcal{J}$ (de todos los elementos de $\mathcal{J}$ que no tienen ni cola de números 0 ni de números $\beta-1$ ) también puede ser visto como el conjunto de todos los elementos de $\mathcal{J}$ que tienen $\beta$-expansión única. Además, se puede notar que todo elemento de $\mathcal{J}$ que posee dos $\beta$-expansiones distintas es un racional, por lo tanto,

$$
\mathcal{J} \backslash \mathbb{Q} \subseteq \mathcal{J} .
$$

Ahora, de manera directa, se tiene que si dos elementos de $\mathcal{J}$ poseen $\beta$-expansiones que coinciden en sus primeras $m$ componentes (con $m \in \mathbb{N}^{*}$ ), entonces la distancia entre los dos elementos es menor o igual que $\frac{1}{\beta^{m-1}}$. Lamentablemente, el recíproco de esto no se cumple en todos los casos. Al igual que antes, para analizar lo expuesto, primero se presenta una propiedad sobre las sucesiones de $\llbracket-\beta+$ $1, \beta+1 \rrbracket$. Lo indicado anteriormente se traduce a que si las primeras $m$ componentes de una sucesión $\left(z_{\mathfrak{n}}\right)_{\mathfrak{n} \in \mathbb{N}}$ de $\llbracket-\beta+1, \beta+1 \rrbracket$ son igual a 0 , entonces

$$
\left|\sum_{n=1}^{+\infty} \frac{z_{n}}{\beta^{n}}\right|<\frac{1}{\beta^{m-1}}
$$


pero su recíproco no es verdadero, por ejemplo, se puede considerar la sucesión definida por

$$
z_{\mathfrak{n}}= \begin{cases}1 & \text { si } n=1 \\ -\beta+1 & \text { si } 2 \leq n<m \\ 0 & \text { si } m \leq n\end{cases}
$$

A pesar de esto, se puede caracterizar este tipo de sucesiones. Se considera el siguiente resultado.

Lema 2 Sea $\left(z_{\mathfrak{n}}\right)_{\mathfrak{n} \in \mathbb{N}}$ una sucesión de $\llbracket-\beta+1, \beta-1 \rrbracket$. Se tiene que si

$$
\left|\sum_{n=1}^{+\infty} \frac{z_{n}}{\beta^{n}}\right|<\frac{1}{\beta}
$$

entonces $z_{1} \in\{-1,0,1\}$. Además, en el caso que $z_{1} \neq 0$, se tiene que, para todo entero $N \geq 2$, si

$$
\left|\sum_{n=1}^{+\infty} \frac{z_{n}}{\beta^{n}}\right|<\frac{1}{\beta^{N}}
$$

entonces, para todo $n \in \llbracket 2, N \rrbracket$,

$$
z_{n}=\left\{\begin{aligned}
\beta-1 & \text { si } z_{1}=-1 \\
-\beta+1 & \text { si } z_{1}=1
\end{aligned}\right.
$$

Demostración. Se supone que

$$
\left|\sum_{n=1}^{+\infty} \frac{z_{n}}{\beta^{n}}\right|<\frac{1}{\beta^{\prime}}
$$

se tiene que

$$
-\frac{1}{\beta}-\frac{z_{1}}{\beta}<\sum_{n=2}^{+\infty} \frac{z_{n}}{\beta^{n}}<\frac{1}{\beta}-\frac{z_{1}}{\beta} .
$$

Además, dado que $\left(z_{\mathfrak{n}}\right)_{\mathfrak{n} \in \mathbb{N}}$ una sucesión de $\llbracket-\beta+1, \beta-1 \rrbracket$, se tiene que

$$
-\frac{1}{\beta}=\sum_{n=2}^{+\infty} \frac{-\beta+1}{\beta^{n}} \leq \sum_{n=2}^{+\infty} \frac{z_{n}}{\beta^{n}} \leq \sum_{n=2}^{+\infty} \frac{\beta-1}{\beta^{n}}=\frac{1}{\beta} .
$$

Así, se tiene que

$$
-\frac{1}{\beta}-\frac{z_{1}}{\beta}<\frac{1}{\beta} \quad y \quad-\frac{1}{\beta}<\frac{1}{\beta}-\frac{z_{1}}{\beta},
$$

de donde, $-2<z_{1}<2$ y, como $z_{1} \in \mathbb{Z}$, concluimos que $z_{1} \in\{-1,0,1\}$.

Ahora, para la segunda parte del resultado, se toma $z_{1} \neq 0$, se utilizará inducción matemática sobre $\mathrm{N} \geq 2$.

- Se supone que

$$
\left|\sum_{n=1}^{+\infty} \frac{z_{n}}{\beta^{n}}\right|<\frac{1}{\beta^{2}} .
$$


Dado que $\frac{1}{\beta^{2}}<\frac{1}{\beta}$, por la parte anterior, se sigue que $z_{1}= \pm 1$. Por lo tanto,

$$
-\frac{1}{\beta^{2}}-\frac{z_{1}}{\beta}-\frac{z_{2}}{\beta^{2}}<\sum_{n=3}^{+\infty} \frac{z_{n}}{\beta^{n}}<\frac{1}{\beta^{2}}-\frac{z_{1}}{\beta}-\frac{z_{2}}{\beta^{2}}
$$

$y$, de nuevo, dado que $\left(z_{n}\right)_{n \in \mathbb{N}}$ una sucesión de $\llbracket-\beta+1, \beta-1 \rrbracket$, se tiene que

$$
-\frac{1}{\beta^{2}}=\sum_{n=3}^{+\infty} \frac{-\beta+1}{\beta^{n}} \leq \sum_{n=3}^{+\infty} \frac{z_{n}}{\beta^{n}} \leq \sum_{n=3}^{+\infty} \frac{\beta-1}{\beta^{n}}=\frac{1}{\beta^{2}},
$$

con esto,

$$
-\frac{1}{\beta^{2}}-\frac{z_{1}}{\beta}-\frac{z_{2}}{\beta^{2}}<\frac{1}{\beta^{2}} \quad \text { y } \quad-\frac{1}{\beta^{2}}<-\frac{1}{\beta^{2}}-\frac{z_{1}}{\beta}-\frac{z_{2}}{\beta^{2}},
$$

de donde

$$
-z_{1} \beta-2<z_{2}<-z_{1} \beta+2 .
$$

De esta forma, si $z_{1}=-1$, entonces $\beta-2<z_{2}<\beta+2$ y, como $z_{1} \in \llbracket-\beta+1, \beta-1 \rrbracket$, se tiene que $z_{2}=\beta-1$; por otro lado, si $z_{1}=1$, de manera análoga, concluimos que $z_{2}=-\beta+1$.

- Se supone que, para un entero $N \geq 2$, se cumple que si

$$
\left|\sum_{n=1}^{+\infty} \frac{z_{n}}{\beta^{n}}\right|<\frac{1}{\beta^{N}}
$$

entonces $z_{n}=-z_{1}(\beta-1)$ para cada $2 \leq n \leq N$. Ahora, se supone que

$$
\left|\sum_{n=1}^{+\infty} \frac{z_{n}}{\beta^{n}}\right|<\frac{1}{\beta^{N+1}}
$$

por lo tanto

$$
-\frac{1}{\beta^{N+1}}+\sum_{n=2}^{N} \frac{z_{1}(\beta-1)}{\beta^{n}}-\frac{z_{N+1}}{\beta^{N+1}}<\sum_{n=N+2}^{+\infty} \frac{z_{n}}{\beta^{n}}<\frac{1}{\beta^{N+1}}+\sum_{n=2}^{N} \frac{z_{1}(\beta-1)}{\beta^{n}}-\frac{z_{N+1}}{\beta^{N+1}},
$$

de donde,

$$
-\frac{1}{\beta^{N+1}}-\frac{z_{1}}{\beta^{N}}-\frac{z_{N+1}}{\beta^{N+1}}<\sum_{n=N+2}^{+\infty} \frac{z_{n}}{\beta^{n}}<\frac{1}{\beta^{N+1}}-\frac{z_{1}}{\beta^{N}}-\frac{z_{N+1}}{\beta^{N+1}}
$$

$\mathrm{y}$, de nuevo como, dado que $\left(z_{\mathfrak{n}}\right)_{\mathrm{n} \in \mathbb{N}}$ una sucesión de $\llbracket-\beta+1, \beta-1 \rrbracket$, se tiene que

$$
-\frac{1}{\beta^{N+1}}=\sum_{n=N+2}^{+\infty} \frac{-\beta+1}{\beta^{n}} \leq \sum_{n=N+2}^{+\infty} \frac{z_{n}}{\beta^{n}} \leq \sum_{n=N+2}^{+\infty} \frac{\beta-1}{\beta^{n}}=\frac{1}{\beta^{N+1}},
$$

con esto

$$
-\frac{1}{\beta^{N+1}}-\frac{z_{1}}{\beta^{N}}-\frac{z_{N+1}}{\beta^{N+1}}<\frac{1}{\beta^{N+1}} \quad y \quad-\frac{1}{\beta^{N+1}}<\frac{1}{\beta^{N+1}}-\frac{z_{1}}{\beta^{N}}-\frac{z_{N+1}}{\beta^{N+1}},
$$

luego

$$
-z_{1} \beta-2<z_{\mathrm{N}+1}<-z_{1} \beta+2 .
$$


Ahora, si $z_{1}=-1$, se sigue que $\beta-2<z_{N+1}<\beta+2$, además, como $z_{N+1} \in \llbracket-\beta+1, \beta-1 \rrbracket$, se concluye que $z_{N+1}=\beta-1$. Por otro lado, de manera análoga, si $z_{1}=1$, se sigue que $z_{N+1}=-\beta+1$.

Ahora, se va a analizar la relación entre la distancia entre dos elementos de $\mathcal{J}$ y la coincidencia de sus $\beta$-expansiones. Es decir, dados dos elementos $a, x \in \mathcal{J}$, con $\beta$-expansiones $\left(a_{n}\right)_{n \in \mathbb{N}}$ y $\left(x_{n}\right)_{n \in \mathbb{N}}$, respectivamente, se formulará condiciones sobre a para que exista $\delta>0$ tal que $|x-a|<\delta$ ( $x$ está suficientemente cerca de a) implique $x_{n}=a_{n}$, para todo $n$ en un intervalo de la forma $\llbracket 1, N \rrbracket$, con algún $N \in \mathbb{N}^{*}$ (sus $\beta$-expansiones coincidan en las primeras componentes).

Primero, se considera el caso en que a no tiene $\beta$-expansión única y se toma $m \in \mathbb{N}^{*}$ como la posición anterior a donde empieza la cola de números 0 o $\beta-1$ de su expansión. Se tiene que, si $x$ está suficientemente cerca de $a$, entonces se puede garantizar la coincidencia de las primeras $m-1$ componentes de sus $\beta$-expansiones. Esto se formaliza en el siguiente resultado.

\section{Proposición 3}

Sea $a \in \mathcal{J}$ tal que posee una $\beta$-expansión $\left(a_{n}\right)_{n \in \mathbb{N}}$ con cola de números 0 . Sea $m \in \mathbb{N}^{*}$ tal que $a_{m} \neq 0$ y $a_{n}=0$ para todo $n>m$. Se tiene que, para cualquier natural $N>m$, existe $\delta>0$ tal que para todo $x \in \mathcal{J}$ con $\beta$-expansión $\left(x_{n}\right)_{n \in \mathbb{N}}$, si

$$
|x-a|<\delta
$$

entonces $x_{n}=a_{n}$ para todo $n \in \llbracket 1, m-1 \rrbracket$. Además,

$$
x_{n}=\left\{\begin{array}{ll}
a_{m}-1 & \text { si } n=m, \\
\beta-1 & \text { si } m<n \leq N
\end{array} \quad \text { o } \quad x_{n}= \begin{cases}a_{m} & \text { si } n=m, \\
0 & \text { si } m<n \leq N .\end{cases}\right.
$$

Demostración. Sea $\mathrm{N}>\mathrm{m}$. Se nota que si

$$
|x-a|=\left|\sum_{n=1}^{+\infty} \frac{x_{n}}{\beta^{n}}-\sum_{n=1}^{m} \frac{a_{n}}{\beta^{n}}\right|<\frac{1}{\beta^{m+1}}
$$

entonces, por el Lema 2, hay tres casos:

$$
x_{1}-a_{1}=-1, \quad x_{1}-a_{1}=0 \quad y \quad x_{1}-a_{1}=1 .
$$

Si $x_{1}-a_{1}=-1$, entonces $x_{m}-a_{m}=\beta-1$; lo cual es absurdo pues $a_{m} \neq 0$. Si $x_{1}-a_{1}=1$, entonces $x_{m+1}=$ $-\beta+1$; lo cual también es absurdo. Se sigue que $x_{1}=a_{1}$. Ahora, de manera similar y procediendo por inducción, se puede demostrar que $x_{n}=a_{n}$ para todo $1 \leq n<m$.

Con esto, se nota que

$$
\begin{aligned}
|x-a|=\left|\sum_{n=1}^{+\infty} \frac{x_{n}}{\beta^{n}}-\sum_{n=1}^{m} \frac{a_{n}}{\beta^{n}}\right| & =\left|\sum_{n=m}^{+\infty} \frac{x_{n}}{\beta^{n}}-\frac{a_{m}}{\beta^{m}}\right| \\
& =\frac{1}{\beta^{m-1}}\left|\sum_{n=m}^{+\infty} \frac{x_{n}}{\beta^{n-m+1}}-\frac{a_{m}}{\beta}\right| \\
& =\frac{1}{\beta^{m-1}}\left|\sum_{n=1}^{+\infty} \frac{x_{m+n-1}}{\beta^{n}}-\frac{a_{m}}{\beta}\right| .
\end{aligned}
$$


De esta forma, si $|x-a|<\frac{1}{\beta^{m+1}}$, entonces

$$
\left|\sum_{n=1}^{+\infty} \frac{x_{m+n-1}}{\beta^{n}}-\frac{a_{m}}{\beta}\right|<\frac{1}{\beta^{2}}
$$

por lo tanto, por el Lema 2, se tiene que $x_{m}-a_{m} \in\{-1,0,1\}$. Se descarta que $x_{m}-a_{m}=1$, pues seguiría que $x_{m+1}=-\beta+1$, por lo tanto, se tienen únicamente dos casos:

- Si $x_{m}=a_{m}$, entonces

$$
\begin{aligned}
|x-a| & =\left|\sum_{n=1}^{+\infty} \frac{x_{n}}{\beta^{n}}-\sum_{n=1}^{m} \frac{a_{n}}{\beta^{n}}\right| \\
& =\left|\sum_{n=m+1}^{+\infty} \frac{x_{n}}{\beta^{n}}\right|=\sum_{n=m+1}^{+\infty} \frac{x_{n}}{\beta^{n}} \\
& =\sum_{n=m+1}^{N} \frac{x_{n}}{\beta^{n}}+\sum_{n=N+1}^{+\infty} \frac{x_{n}}{\beta^{n}} .
\end{aligned}
$$

De esta forma, si $|x-a|<\frac{1}{\beta^{N}}$, entonces

$$
\sum_{n=m+1}^{N} \frac{x_{n}}{\beta^{n}} \leq \sum_{n=m+1}^{N} \frac{x_{n}}{\beta^{n}}+\sum_{n=N+1}^{+\infty} \frac{x_{n}}{\beta^{n}}<\frac{1}{\beta^{N}}
$$

es decir

$$
\sum_{n=m+1}^{N} x_{n} \beta^{N-n}<1
$$

de donde $x_{n}=0$ para todo $m<n \leq N$ pues la suma consta solo de números naturales.

- Si $x_{m}=a_{m}-1$, entonces,

$$
|x-a|=\left|\frac{-1}{\beta^{m}}+\sum_{n=m+1}^{+\infty} \frac{x_{n}}{\beta^{n}}\right|=\frac{1}{\beta^{m-1}}\left|\frac{-1}{\beta}+\sum_{n=2}^{+\infty} \frac{x_{n+m-1}}{\beta^{n}}\right| .
$$

De esta manera, si $|x-a|<\frac{1}{\beta^{N}}$, entonces,

$$
\left|\frac{-1}{\beta}+\sum_{n=2}^{+\infty} \frac{x_{n+m-1}}{\beta^{n}}\right|<\frac{1}{\beta^{N-m+1}},
$$

por lo tanto, por el Lema $2, x_{n+m-1}=\beta-1$ para todo $n \in \llbracket 2, N-m+1 \rrbracket$, es decir, $x_{n}=\beta-1$ para todo $m<n \leq N$.

Por todo lo anterior, se sigue que el resultado es verdadero si se toma

$$
\delta=\min \left\{\frac{1}{\beta^{m+1}}, \frac{1}{\beta^{N}}\right\}=\frac{1}{\beta^{N}} .
$$

Por otro lado, si se considera el caso en que a tiene una única $\beta$-expansión, se tiene que, se puede garantizar siempre la coincidencia de una cantidad cualquiera de las primeras componentes de las $\beta$-expansión de a y $x$, siempre que $x$ esté suficientemente cerca de a. El siguiente resultado formaliza esta idea. 


\section{Proposición 4}

Sea $a \in \mathcal{J}$ con $\beta$-expansión $\left(a_{n}\right)_{n \in \mathbb{N}}$. Se tiene que, para cualquier $N \in \mathbb{N}^{*}$, existe $\delta>0$ tal que para todo $x \in \mathcal{J}$ con $\beta$-expansión $\left(x_{n}\right)_{n \in \mathbb{N}}$, si

$$
|x-a|<\delta
$$

entonces $x_{n}=a_{n}$ para todo $n \in \llbracket 1, N \rrbracket$.

Demostración. Primero, se observa que, $\left(a_{n}\right)_{n \in \mathbb{N}}$ no posee cola de números 0 ni de número $\beta-1$, pues a tiene $\beta$-expansión única. Ahora se demuestra el resultado por inducción finita.

- Para $N=1$, se toma $k \geq 2$ tal que $a_{k} \notin\{0, \beta-1\}$, lo cual es posible por la forma de las colas de $\left(a_{n}\right)_{n \in \mathbb{N}}$. Se nota que si

$$
|x-a|<\frac{1}{\beta^{k}}
$$

entonces

$$
\left|\sum_{n=1}^{+\infty} \frac{x_{n}-a_{n}}{\beta^{n}}\right|<\frac{1}{\beta^{k}}
$$

Ahora, si se supone que $x_{1} \neq y_{1}$, por el Lema 2, se sigue que $x_{k}-a_{n} \in\{\beta+1, \beta-1\}$, lo cual es imposible por la forma en que está tomado $a_{k}$, por lo tanto, $x_{1}=a_{1}$. Así, en este caso es suficiente tomar $\delta=\frac{1}{\beta^{\mathrm{k}}}$ para tener el resultado.

- Sea $N \geq 1$, se supone que existe $\delta_{N}>0$ tal que si

$$
|x-a|<\delta
$$

entonces $x_{n}=a_{n}$ para todo $n \in \llbracket 1, N \rrbracket$. Se demuestra que el resultado es verdadero para $N+1$.

De manera análoga al caso anterior, se toma $k \geq 2$ tal que $a_{N+k} \notin\{0, \beta-1\}$. Se nota que si

$$
|x-a|<\min \left\{\delta_{N}, \frac{1}{\beta^{N+K}}\right\} \text {, }
$$

entonces

$$
\left|\sum_{n=1}^{+\infty} \frac{x_{n}-a_{n}}{\beta^{n}}\right|=\left|\sum_{n=N+1}^{+\infty} \frac{x_{n}-a_{n}}{\beta^{n}}\right|=\frac{1}{\beta^{N}}\left|\sum_{n=1}^{+\infty} \frac{x_{N+n}-a_{N+n}}{\beta^{n}}\right|<\frac{1}{\beta^{N+k}},
$$

por lo tanto

$$
\left|\sum_{n=1}^{+\infty} \frac{x_{N+n}-a_{N+n}}{\beta^{n}}\right|<\frac{1}{\beta^{k}} .
$$

Así, de manera análoga al paso anterior, utilizando el Lema 2, se concluye que $x_{N+1}=a_{N+1}$. Por lo tanto, en este caso es suficiente tomar $\delta=\operatorname{mín}\left\{\delta_{N}, \frac{1}{\beta^{N+K}}\right\}$ para obtener el resultado. 


\section{Continuidad de funciones dadas por reordenaciones}

Para generalizar las funciones presentadas en las ecuaciones (1), se considera la siguiente notación, en adelante: dada $\psi: \mathbb{N}^{*} \rightarrow \mathbb{N}^{*}$ una función (a la cual se la llamará un reordenamiento), definimos

$$
\begin{aligned}
F_{\psi}: \mathcal{J} & \longrightarrow \mathcal{J} \\
x & \longmapsto \sum_{n=1}^{+\infty} \frac{E_{\psi(n)}^{\beta}(x)}{\beta^{n}}=\sum_{n=1}^{+\infty} \frac{E_{\psi(n)}(x)}{\beta^{n}} .
\end{aligned}
$$

De esta forma, si $\psi: \mathbb{N}^{*} \rightarrow \mathbb{N}^{*}$, para $x \in \mathcal{J}$, con $\beta$-expansión $\left(x_{\mathfrak{n}}\right)_{\mathfrak{n} \in \mathbb{N}}$ sin cola de términos $\beta-1$, se tiene que

$$
\begin{aligned}
F_{\psi}(x) & =\sum_{n=1}^{+\infty} \frac{E_{\psi(n)}(x)}{\beta^{n}}=\sum_{n=1}^{+\infty} \frac{x_{\psi(n)}}{\beta^{n}} \\
& =\frac{x_{\psi(1)}}{2^{1}}+\frac{x_{\psi(2)}}{2^{2}}+\frac{x_{\psi(3)}}{2^{3}}+\frac{x_{\psi(4)}}{2^{4}}+\cdots
\end{aligned}
$$

Se puede apreciar que esta función es la formalización de idea de "reordenar una $\beta$-expansión de un número". Además, se recuerda que si $x \in \mathcal{J}$, entonces $\left(E_{n}(x)\right)_{n \in \mathbb{N}}$ es una $\beta$-expansión de $x$ que no posee cola de números $\beta-1$.

A continuación, se estudia la continuidad de este tipo de funciones utilizando algunos resultados de la sección precedente. Se tiene que estas funciones son continuas en todo elemento que tenga $\beta$ expansión única.

\section{Proposición 5}

Sea $\psi: \mathbb{N}^{*} \rightarrow \mathbb{N}^{*}$ una función. Se tiene que $F_{\psi}$ es continua en $\mathcal{J}$.

Demostración. Sea $a \in \mathcal{J} y \in>0$. Se nota que, para cada $x \in \mathcal{J}$ y cada entero $N \geq 1$,

$$
\begin{aligned}
\left|F_{\psi}(x)-F_{\psi}(a)\right| & =\left|\sum_{n=1}^{+\infty} \frac{E_{\psi(n)}(x)-E_{\psi(n)}(a)}{\beta^{n}}\right| \\
& \leq \sum_{n=1}^{N} \frac{\left|E_{\psi(n)}(x)-E_{\psi(n)}(a)\right|}{\beta^{n}}+\sum_{n=N+1}^{+\infty} \frac{\left|E_{\psi(n)}(x)-E_{\psi(n)}(a)\right|}{\beta^{n}} \\
& <\sum_{n=1}^{N} \frac{\left|E_{\psi(n)}(x)-E_{\psi(n)}(a)\right|}{\beta^{n}}+\frac{1}{\beta^{N}} .
\end{aligned}
$$

Por lo tanto, si se toma $N \in \mathbb{N}^{*}$ tal que $\frac{1}{\beta^{N}} \leq \epsilon$, por la Proposición 4 , para $M=\operatorname{máx}\{\psi(n): 1 \leq n \leq N\}$, existe $\delta>0$ tal que, para todo $x \in \mathcal{J},|x-a|<\delta$ implica que $E_{m}(x)=E_{m}(a)$ para todo $1 \leq m \leq M$, de donde, $E_{\psi(n)}(x)=E_{\psi(n)}(a)$ para todo $1 \leq n \leq N$. Por lo tanto, si $|x-a|<\delta$, entonces

$$
\left|F_{\psi}(x)-F_{\psi}(a)\right|<\sum_{n=1}^{N} \frac{\left|E_{\psi(n)}(x)-E_{\psi(n)}(a)\right|}{\beta^{n}}+\frac{1}{\beta^{N}} \leq \epsilon .
$$

Por lo tanto, $F_{\psi}$ es continua en a. Así, se tiene que $F_{\psi}$ es continua en $\mathcal{J}$.

Con esto, se tiene de manera directa el resultado principal de este trabajo. 


\section{Teorema 1}

Sea $\psi: \mathbb{N}^{*} \rightarrow \mathbb{N}^{*}$ una función. Se tiene que $\mathrm{F}_{\psi}$ es continua en casi todas partes $\mathrm{y}$, por lo tanto, una variable aleatoria.

Demostración. Sea $\mathrm{C}$ el conjunto de puntos donde $\mathrm{F}_{\phi}$ es continua, por el resultado anterior, se tiene que $\mathcal{J} \subseteq C$, además, por la expresión (4), se sigue que $\mathcal{J} \backslash \mathbb{Q} \subseteq C$, por lo tanto, $C$ tiene medida 1 , así, el conjunto de puntos donde $F_{\psi}$ no es continua tiene medida 0 , de donde, $F_{\psi}$ es continua en casi todas partes y, por la completitud de la medida de Lebesgue (Bartle, 1995), se tiene que es una variable aleatoria.

Luego de este resultado, cabe la pregunta si se tiene que las funciones $F_{\psi}$ son continuas en todo J. En lo que sigue de esta sección se analizará esto.

Como se puede observar, la Proposición 5 depende la Proposición 4. Dado que el lema no puede aplicarse a todo J, no se puede realizar una extensión directa del resultado de la Proposición 5 para $\mathcal{J} \backslash \mathcal{J}$. Es más, este tipo de funciones pueden tener discontinuidades. El siguiente resultado muestra características de puntos donde estas funciones no son continua.

\section{Proposición 6}

Sea $\psi: \mathbb{N}^{*} \rightarrow \mathbb{N}^{*}$ una función. Si $m \in \mathbb{N}^{*}$ es tal que $m \notin \operatorname{img}(\psi)$ y $\left.\operatorname{img}(\psi) \cap\right] m,+\infty[\neq \varnothing$, entonces, para todo $a_{1}, \ldots, a_{m} \in \llbracket 0, \beta-1 \rrbracket$ con $a_{m} \neq 0$, se tiene que $F_{\psi}$ no es continua en

$$
a=\sum_{n=1}^{m} \frac{a_{n}}{\beta^{n}} .
$$

Demostración. Se supone que $m \in \mathbb{N}^{*}$ es tal que $m \notin \operatorname{img}(\psi)$ y $\left.\operatorname{img}(\psi) \cap\right] m,+\infty\left[\neq \varnothing\right.$. Sean $a_{1}, \ldots, a_{m} \in$ $\llbracket 0, \beta-1 \rrbracket$ con $a_{m} \neq 0$ y se define

$$
a=\sum_{n=1}^{m} \frac{a_{n}}{\beta^{n}} .
$$

Se toma $M \in \mathbb{N}^{*}$ tal que $\psi(M)>m$. Dado $\delta>0$, existe un entero $N \geq \psi(M)$ tal que

$$
\frac{1}{\beta^{N}}<\delta
$$

Ahora, tomando $x \in \mathcal{J}$ tal que

$$
E_{n}(x)= \begin{cases}a_{n} & \text { si } 1 \leq n<m \\ a_{m}-1 & \text { si } n=m \\ \beta-1 & \text { si } m<n \leq N \\ 0 & \text { si } N<n\end{cases}
$$

se tiene que

$$
\begin{aligned}
|x-a| & =\left|\sum_{n=1}^{+\infty} \frac{E_{n}(x)}{\beta^{n}}-\sum_{n=1}^{m} \frac{a_{n}}{\beta^{n}}\right|=\left|-\frac{1}{\beta^{m}}+\sum_{n=m+1}^{N} \frac{\beta-1}{\beta^{n}}\right| \\
& =\left|-\frac{1}{\beta^{m}}+\frac{1}{\beta^{m}}-\frac{1}{\beta^{N}}\right|=\frac{1}{\beta^{N}}<\delta .
\end{aligned}
$$


Por otro lado, dado que $m \notin i m g(\psi)$, se nota que

$$
F_{\psi}(a)=\sum_{n=1}^{+\infty} \frac{E_{\psi(n)}(a)}{\beta^{n}}=\sum_{\substack{n=1 \\ 1 \leq \psi(n)<m}}^{+\infty} \frac{a_{\psi(n)}}{\beta^{n}}
$$

además

$$
F_{\psi}(x)=\sum_{n=1}^{+\infty} \frac{E_{\psi(n)}(x)}{\beta^{n}}=\sum_{\substack{n=1 \\ 1 \leq \psi(n)<m}}^{+\infty} \frac{a_{\psi(n)}}{\beta^{n}}+\sum_{\substack{n=1 \\ m<\psi(n) \leq N}}^{+\infty} \frac{\beta-1}{\beta^{n}} .
$$

De esta forma, puesto que $m<\psi(M) \leq N$,

$$
\left|F_{\psi}(x)-F_{\psi}(a)\right|=\left|\sum_{\substack{n=1 \\ m<\psi(n) \leq N}}^{+\infty} \frac{\beta-1}{\beta^{n}}\right|=\sum_{\substack{n=1 \\ m<\psi(n) \leq N}}^{+\infty} \frac{\beta-1}{\beta^{n}} \geq \frac{\beta-1}{\beta^{M}}>0 .
$$

Luego, $F_{\psi}$ no es continua en $a$.

El resultado anterior indica que las funciones $F_{\psi}$, bajo hipótesis adecuadas, no son continuas en elementos de $\mathcal{J}$ que tienen una forma concreta, de hecho, esta forma implica que el elemento tiene una $\beta$-expansión con cola de números 0 y, por lo tanto, no tiene $\beta$-expansión única. La pregunta, ahora, recaería en analizar si es siempre discontinua en puntos son $\beta$-expansión única. En general, esto no es así, dado que se puede extender, de cierta manera, el resultado de la Proposición 4 a puntos sin $\beta$-expansión única mediante la Proposición 3, se tiene el siguiente resultado que caracteriza los elementos de $\mathcal{J} \backslash \mathcal{J}$ en los cuales las funciones $F_{\psi}$, son continuas.

\section{Proposición 7}

Sea $\psi: \mathbb{N}^{*} \rightarrow \mathbb{N}^{*}$ una función estrictamente creciente. Si $m \in \mathbb{N}^{*}$ es tal que $m \in \operatorname{img}(\psi)$, entonces, para todo $a_{1}, \ldots, a_{m} \in \llbracket 0, \beta-1 \rrbracket$ con $a_{m} \neq 0$, se tiene que $F_{\psi}$ es continua en

$$
a=\sum_{n=1}^{m} \frac{a_{n}}{\beta^{n}}
$$

Demostración. Se supone que $m \in \mathbb{N}^{*}$ es tal que $m \in \operatorname{img}(\psi)$. Sean $a_{1}, \ldots, a_{m} \in \llbracket 0, \beta-1 \rrbracket$, con $a_{m} \neq 0$, y se define

$$
a=\sum_{n=1}^{m} \frac{a_{n}}{\beta^{n}}
$$

Ahora, sea $\epsilon>0$. Como $m \in \operatorname{img}(\psi)$, se toma $k \in \mathbb{N}^{*}$ tal que $m=\psi(k)$. Se toma $N \in \mathbb{N}^{*}$ tal que

$$
\frac{1}{\beta^{N}}<\frac{\epsilon}{2}
$$

Por la Proposición 3, para $\psi(N) \in \mathbb{N}^{*}$, existe $\delta>0$ tal que, para todo $x \in \mathcal{J},|x-a|<\delta$ implica que $\mathrm{E}_{\mathrm{n}}(\mathrm{x})=\mathrm{E}_{\mathrm{n}}(\mathrm{a})$ para todo $1 \leq \mathrm{n}<\mathrm{m}$, además,

$$
E_{n}(x)=\left\{\begin{array}{ll}
E_{m}(a)-1 & \text { si } n=m, \\
\beta-1 & \text { si } m<n \leq \psi(N),
\end{array} \quad \text { o } \quad E_{n}(x)= \begin{cases}E_{m}(a) & \text { si } n=m, \\
0 & \text { si } m<n \leq \psi(N) .\end{cases}\right.
$$


De aquí, se tiene que, para todo $x \in \mathcal{J}$, si $|x-a|<\delta$, entonces

$$
E_{\psi(n)}(x)=\left\{\begin{array}{ll}
E_{\psi(n)}(a) & \text { si } 1 \leq n<k \\
E_{\psi(k)}(a)-1 & \text { si } n=k, \\
\beta-1 & \text { si } k<n \leq N
\end{array} \quad \text { o } \quad E_{\psi(n)}(x)= \begin{cases}E_{\psi(n)}(a) & \text { si } 1 \leq n<k \\
E_{\psi(k)}(a) & \text { si } n=k \\
0 & \text { si } k<n \leq N\end{cases}\right.
$$

De esta forma, se tiene que para todo $x \in \mathcal{J}$, si $|x-a|<\delta$, entonces se tienen dos casos.

- Considerando el primer caso, se tiene que

$$
\begin{aligned}
\left|F_{\psi}(x)-F_{\psi}(a)\right| & =\left|\sum_{n=1}^{+\infty} \frac{E_{\psi(n)}(x)}{\beta^{n}}-\sum_{n=1}^{m} \frac{E_{\psi(n)}(a)}{\beta^{n}}\right| \\
& =\left|-\frac{1}{\beta^{k}}+\sum_{n=k+1}^{N} \frac{\beta-1}{\beta^{n}}+\sum_{n=N+1}^{+\infty} \frac{E_{\psi(n)}(x)}{\beta^{n}}\right| \\
& =\left|-\frac{1}{\beta^{k}}+\frac{1}{\beta^{k}}-\frac{1}{\beta^{N}}+\sum_{n=N+1}^{+\infty} \frac{E_{\psi(n)}(x)}{\beta^{n}}\right| \\
& \leq \frac{1}{\beta^{N}}+\sum_{n=N+1}^{+\infty} \frac{E_{\psi(n)}(x)}{\beta^{n}} \leq \frac{1}{\beta^{N}}+\sum_{n=N+1}^{+\infty} \frac{\beta-1}{\beta^{n}} \\
& =\frac{1}{\beta^{N}}+\frac{1}{\beta^{N}}<\epsilon .
\end{aligned}
$$

- Considerando el segundo caso, se tiene que

$$
\begin{aligned}
\left|F_{\psi}(x)-F_{\psi}(a)\right| & =\left|\sum_{n=1}^{+\infty} \frac{E_{\psi(n)}(x)}{\beta^{n}}-\sum_{n=1}^{m} \frac{E_{\psi(n)}(a)}{\beta^{n}}\right| \\
& =\left|\sum_{n=N+1}^{+\infty} \frac{E_{\psi(n)(x)}}{\beta^{n}}\right| \\
& =\sum_{n=N+1}^{+\infty} \frac{E_{\psi(n)(x)}}{\beta^{n}} \leq \sum_{n=N+1}^{+\infty} \frac{\beta-1}{\beta^{n}} \\
& \leq \frac{1}{\beta^{N}}<\epsilon .
\end{aligned}
$$

Así, se sigue que $F_{\psi}$ es continua en $a$.

Con los resultados precedentes, se logra caracterizar completamente, de manera directa, los puntos de discontinuidad de las funciones $F_{\psi}$, cuando $\psi$ es una función estrictamente creciente. Así, se tiene el siguiente resultado.

\section{Teorema 2}

Si $\psi: \mathbb{N}^{*} \rightarrow \mathbb{N}^{*}$ es una función estrictamente creciente, el conjunto de discontinuidades de $F_{\psi}$ es:

$$
\bigcup_{m \notin i m g(\psi)}\left\{x \in \mathcal{J}: E_{m}(x) \neq 0 y E_{n}(x)=0 \text { para todo } n>m\right\}
$$




\section{Conclusiones}

Se presentó de manera explícita, directa y original una demostración al hecho de que dada una función $\psi: \mathbb{N}^{*} \rightarrow \mathbb{N}^{*}$, la función $F_{\psi}$ es continua en casi todas partes. Para esto, se presentaron algunos resultados de las $\beta$-expansiones que, aunque son conocidos, las demostraciones aquí realizadas no utilizan resultados fuertes de áreas como la Teoría de la medida o la Teoría de la probabilidad, como lo hacen Billingsley (2012); Kallenberg (2002); Williams (1991), sino que se desarrolla basándose únicamente en las definiciones. Entre estos resultados están:

1. Dado que todo número en $\mathcal{J}$ tiene una $\beta$-expansión y como para toda $x \in \mathbb{R}, x-\lfloor x\rfloor \in[0,1[$, se tiene que todo número real tiene al menos una $\beta$-expansión y a lo sumo dos.

2. Las $\beta$-expansiones de números "cercanos" coinciden en sus primeras componentes. En el caso de que un número tenga $\beta$-expansión única, para cualquier índice, se puede determinar un radio $r>0$, suficientemente pequeño, para que los números con distancia a lo más $r$ tengan la misma $\beta$-expansión hasta ese índice.

3. Sabiendo que todo número irracional tiene $\beta$-expansión única, se tiene que $\mathcal{J}$, el conjunto de números con $\beta$-expansión única, tiene medida 1.

El Teorema 1, justifica completa y detalladamente que las funciones presentadas por Billingsley (2012); Kallenberg (2002); Williams (1991), son, en efecto, variables aleatorias, es más, son funciones continuas en casi todas partes. La demostración de este teorema es original y plantea una forma constructiva de generar este tipo de funciones; es decir, se tiene una forma constructiva de generar variables aleatorias.

Por otro lado, como implicación del Teorema 1, se tiene que, partiendo de las funciones dadas por los reordenamientos del zigzag de Cantor:

$$
\begin{aligned}
\psi_{m}: \mathbb{N} & \longrightarrow \mathbb{N} \\
n & \longmapsto \frac{n^{2}+(2 m-1) n+m^{2}-3 m+2}{2},
\end{aligned}
$$

para todo $m \in \mathbb{N}$, se pueden generar las funciones $\varphi_{m}=F_{\psi_{m}}$ (en referencia a las funciones definidas por las ecuaciones (1)) y, por lo tanto, también son variables aleatorias, así, las funciones presentadas en las ecuaciones (1) son variables aleatorias, completando de esta manera la demostración dada por Williams (1991).

Finalmente, la Proposición 7 da un paso más allá y establece que si la reordenación es "creciente", entonces los puntos de discontinuidad de las funciones quedan completamente caracterizados bajo el resultado del Teorema 2.

\section{Referencias}

Bartle, R. G. (1995). The elements of integration and lebesgue measure. New York: John Wiley \& Sons. Billingsley, P. (2012). Probability and measure. Estados Unidos de América: John Wiley \& Sons, Inc. Borovkov, A. (2013). Probability theory. Londres: Springer-Verlag.

Chung, K., y Zhong, K. (2001). A course in probability theory. Estados Unidos de América: Elsevier Science.

Gentili, S. (2020). Measure, integration and a primer on probability theory. Suiza: Springer.

Kallenberg, O. (2002). Fundations of modern probability. Estados Unidos de América: Springer. 
Rényi, A. (1957). Representations for real numbers and their ergodic properties. Acta Mathematica Academiae Scientiarum Hungarica, 8(3), 477-493.

Shirali, S. (2018). A concise introduction to measure theory. Suiza: Springer.

Williams, D. (1991). Probability with martingales. New York: Cambridge University Press. 